\title{
USO DE ANTICORPOS MONOCLONAIS NO TRATAMENTO DO CÂNCER DE MAMA: UMA REVISÃO DA LITERATURA
}

\author{
USE OF MONOCLONAL ANTIBODIES IN THE TREATMENT OF BREAST CANCER. A \\ LITERATURE REVIEW
}

\author{
Maria Carolina Epifânio Pimentel ${ }^{1}$ \\ Claudinei Mesquita da Silva ${ }^{2}$ \\ Leyde Daiane de Peder ${ }^{3}$
}

RESUMO: A ação dos anticorpos monoclonais tem fomentado o segmento científico, gerando resultados promissores e dúvidas quanto sua usualidade em certas neoplasias. $O$ objetivo do estudo foi analisar através de uma revisão da literatura, o uso de mAbs pode, efetivamente, auxiliar no tratamento do câncer de mama. Revisão da literatura em bases de dados eletrônicos: Literatura Latino-Americana e do Caribe em Ciências da Saúde (LILAC'S), MEDLINE, National Center for Biotechnology Information (NCBI), National Library of Medicine (PubMed) e Scientific Electronic Library Online (SCIELO). Os estudos selecionados relatam que a nova classe de biofármacos é detentora de perspectiva de inovação para o sucesso no tratamento oncológico comparada a agentes citotóxicos e da radioterapia.

Palavras-chave: Anticorpos Monoclonais. Câncer de Mama. Tratamento.

\footnotetext{
I Pedagogia - Universidade Estadual do Oeste do Paraná - Unioeste. Pós em Educação Infantil - Unopar. Farmácia- Centro Universitário Assis Gurgacz.Acadêmica do Curso de Farmácia do Centro Universitário Fundação Gurgacz, Cascavel, Paraná, Brasil. E-mail:_carolinapimentel2502@hotmail.com .Orcid: oooo-ooor7385-0995.

${ }^{2}$ Graduado em Farmácia com Habilitação em Análises Clinicas pela Universidade Estadual de Maringá (I997200I). Mestre em Biologia Molecular, com dissertação na área de bioquímica (2002-2004) pela Universidade Federal de São Paulo (UNIFESP), e Doutor em Ciências da Saúde, com tese na área de Doenças Infecciosas e Parasitárias pela Universidade Estadual de Maringá (2015-2018). Atualmente é professor do núcleo de Química e Análises Clínicas do Centro Universitário da Fundação Assis Gurgacz. Tem projetos de pesquisa vinculados às áreas de análises clínicas e toxicológicos, onde exerce o cargo de líder de pesquisa. Doutor em Ciências da Saúde, Centro Universitário da Fundação Assis Gurgacz, Cascavel, Paraná, Brasil. E-mail: claudinei@fag.edu.br. Orcid: oooo-ooo3-4393-0331.

${ }^{3}$ Graduação em Farmácia e Bioquímica pela Universidade Estadual de Maringá (2003), mestrado em Ciências pela Universidade Federal de São Paulo-Escola Paulista de Medicina (2006) e é Doutora pelo Programa de Biociências e Fisiopatologia da Universidade Estadual de Maringá (2018). Atualmente é professora do curso de Farmácia do Centro Universitário da Fundação Assis Gurgacz e é fiscal designada na Vigilância Sanitária da Prefeitura Municipal de Cascavel. Doutora em Fisiopatologia, Centro Universitário da Fundação Assis Gurgacz, Cascavel, Paraná, Brasil. E-mail: leydepeder@yahoo.com.br.Orcid:oooo-ooo2-o814-2586.
} 
ABSTRACT: The monoclonal antibodies' (mAbs) action has instigated the scientific segment, generating promising results and doubts analyzing its usuality in determined neoplasms. The main purpose of this research was to investigate through a literature review, if the mAbs usage can, effectively, assist on breast cancer treatment. Literature review based on electronic data: Literatura Latino-Americana e do Caribe em Ciências da Saúde (LILAC'S), MEDLINE, National Center for Biotechnology Information (NCBI), National Library of Medicine (PubMed) and Scientific Electronic Library Online (SCIELO). The selected research report that the new biopharmaceuticals' group holds the perspective of innovation to success in oncological treatment compared to cytotoxic agents and to radiotherapy ones.

Keywords: Monoclonal Antibodies. Breast Cancer. Treatment.

\section{INTRODUÇÃO}

Câncer de mama, no Brasil, é o tipo de câncer mais comum entre as mulheres e o segundo mais frequente (INCA, 2020). Diante de um cenário contemporâneo e o avanço das pesquisas clínicas, destaca-se dentre outras formas de tratamento, as mais promissoras e positivas, que envolvem o emprego das tecnologias citotóxicas inovadoras da inibição de angiogênese, tais quais os anticorpos monoclonais ( $\mathrm{mAbs}$ ). $\mathrm{O}$ tratamento para o câncer com medicamentos biológicos é recente, se comparado aos tratamentos com os medicamentos produzidos por síntese química. Tais expectativas motivaram o desejo por desenvolver estudos que envolvam a utilização de mAbs na remissão e possível cura do câncer de mama (CARVALHO, 2013).

Os mAbs são imunoglobulinas produzidas por linhas celulares híbridas (hibridomas) derivadas da fusão clonal de linfócitos B; sendo os mesmos modulados a dispor reação junto a antígenos específicos (seletividade) a dispor normalização da circulação existente e, em especial, o cerceamento (inibição) da angiogênese vascular, vide o reconhecimento e ligação dessas ao fator de crescimento endotelial vascular (internacionalmente conhecido pela sigla VEGF), o que torna o VEGF incapaz de ativar seu receptor e assim gerar novos vasos sanguíneos (CARVALHO, 2013).

Tais atos permitem que o tumor não seja mais nutrido bem como possibilita que medicamentos quimioterápicos empregados ao tratamento possam agir com uma maior eficácia no tumor, destruindo, bem como retardando seu crescimento e disseminação descontrolada. O fato é que a ação dos mAbs tem fomentado o segmento científico, gerando 
muita curiosidade, bem como dúvidas, principalmente, quanto a sua usualidade desses junto a tipos de câncer específicos, tais como o de mama (COELHO, 20r4; PEÑA et al., 2014; VIDAL et al., 2018; ZIDAN, 2020). Assim, o presente estudo busca analisar se o uso de mAbs pode, efetivamente, auxiliar no tratamento do câncer de mama. Além disso, buscou-se elucidar os principais fatores de risco, fisiopatologia, diagnóstico, classificação, rastreio e tratamento da patologia.

\section{METODOLOGIA}

Revisão da literatura, utilizando livros e periódicos buscados em cinco bases de dados eletrônicos: Literatura Latino-Americana e do Caribe em Ciências da Saúde (LILAC'S), MEDLINE, National Center for Biotechnology Information (NCBI), National Library of Medicine (PubMed) e Scientific Electronic Library Online (SCIELO).

A coleta de dados deu-se entre Fevereiro a Julho de 2021, empregando os seguintes Descritores em Ciências da Saúde (DeCS): câncer de mama e anticorpos monoclonais. O acrônimo associado as palavras chaves foi AND. Os critérios de inclusão definidos foram: artigos e/ou estudos publicados entre 2009 e 2020, que detenham entendimentos relacionados à elucidação de como se dá o uso de mAbs na cura do câncer de mama, com conteúdo em português, inglês ou espanhol, e que estejam indexados nas bases de dados supracitados

Os artigos foram analisados e distribuídos entre os autores para leitura independente, seguida de validação por consenso. Os autores também analisaram o conteúdo extraído de cada artigo individualmente e inserido na Tabela I, sendo que eventuais discrepâncias nos resultados descritos foram resolvidas por consenso.

Foram extraídas as seguintes variáveis de todos os estudos elegíveis como: predominância no sexo feminino, independentemente da faixa etária, apresenta-se em etnias brancas, sem distinção de classe social, sedentárias, com antecedente familiar de câncer de mama. A aprovação ética não foi necessária para este estudo, pois foi baseado em dados / informações recuperadas de estudos publicados já disponíveis no domínio público.

\section{RESULTADOS E DISCUSSÃO}

$\mathrm{Na}$ base LILACS, utilizando as palavras-chaves câncer de mama and anticorpos monoclonais, foram selecionados 727 artigos. Após a inserção dos filtros - texto completo, 
assuntos principais (neoplasias de mama, antineoplásticos, fator A de crescimento de endotélio vascular), tipo de estudo (ensaio clínico controlado), idiomas (inglês e espanhol), período de tempo (2009 a 2020) foram selecionados 84 estudos. Após a leitura dos títulos e resumos, foram incluídos 13 artigos para leitura na integra.

Na base MEDLINE, utilizando os termos anticorpo monoclonal and câncer de mama, anticorpos monoclonais e tratamento, foram encontrados 201 estudos relacionados. Após leitura de título e resumo, foram selecionados I2 artigos para análise posterior.

Já base NCBI, foram apresentados 279, utilizando os DECS câncer de mama e anticorpos monoclonal. A pós aplicação de filtros pertinentes ao status do estudo (concluído), tipo de estudo (intervencional) e estudos com que apresentassem resultados, restaram 8I artigos. Destes, 9 foram selecionados para leitura na íntegra.

Na PubMed a busca apresentou 2.594 artigos, utilizando como DECS câncer de mama e anticorpos monoclonais acerca da temática abordada. Após aplicação dos filtros - tempo (2009 a 2020), texto completo grátis, tipo de artigo (livros e documentos, ensaio clínicos, teste controlado e aleatório). Restaram i66 artigos, os quais, is foram selecionados para leitura.

Por fim, a busca na plataforma SCIELO utilizando as palavras chaves câncer de mama and. anticorpos monoclonais resultaram em io artigos, destes, 3 foram selecionados para posterior leitura.

Ao final foram selecionados 52 estudos e dentre estes, 5 contemplaram os todos os critérios de seleção (Figura I). Os estudos selecionados, juntamente com o nome do primeiro autor, título, objetivos e principais resultados estão ilustrados a seguir na Tabela I.

Câncer é uma patologia onde a população celular cresce e/ ou se divide sem respeitar os limites fisiológicos, ditos normais, invadindo e destruindo tecidos vizinhos/ adjacentes, e se espalhando a diversos locais de um mesmo corpo/ organismo por meio do processo de metástase. É interessante lembrar que o câncer (I) dito benigno se mostra autolimitado, sendo referida limitação, comumente, utilizada a diferenciar um câncer benigno de um câncer maligno, e (2) indiferente de ser o câncer benigno ou maligno, pode esse afetar indivíduos de todas as idades, no entanto quanto maior a idade maior o risco (ALVES et al., 2010; FIGUEIREDO, 2013; COELHO, 2014; OPAS, 2018). 
Para indivíduos do sexo feminino, o câncer de mama é um dos mais temidos, visto que esse, dentre outros atos, desregula na mulher toda sua percepção de sexualidade e autoimagem (ALVES et al., 2010; LEAL et al., 2010; SANTOS et al., 201I). No Brasil, é o câncer de mama um dos que mais comumente afeta as mulheres; tendo esses o índice de 13,6\% de mortalidade pós diagnósticos (WHO, 2019).

Figura I. Diagrama de fluxo dos estudos selecionados para revisão.

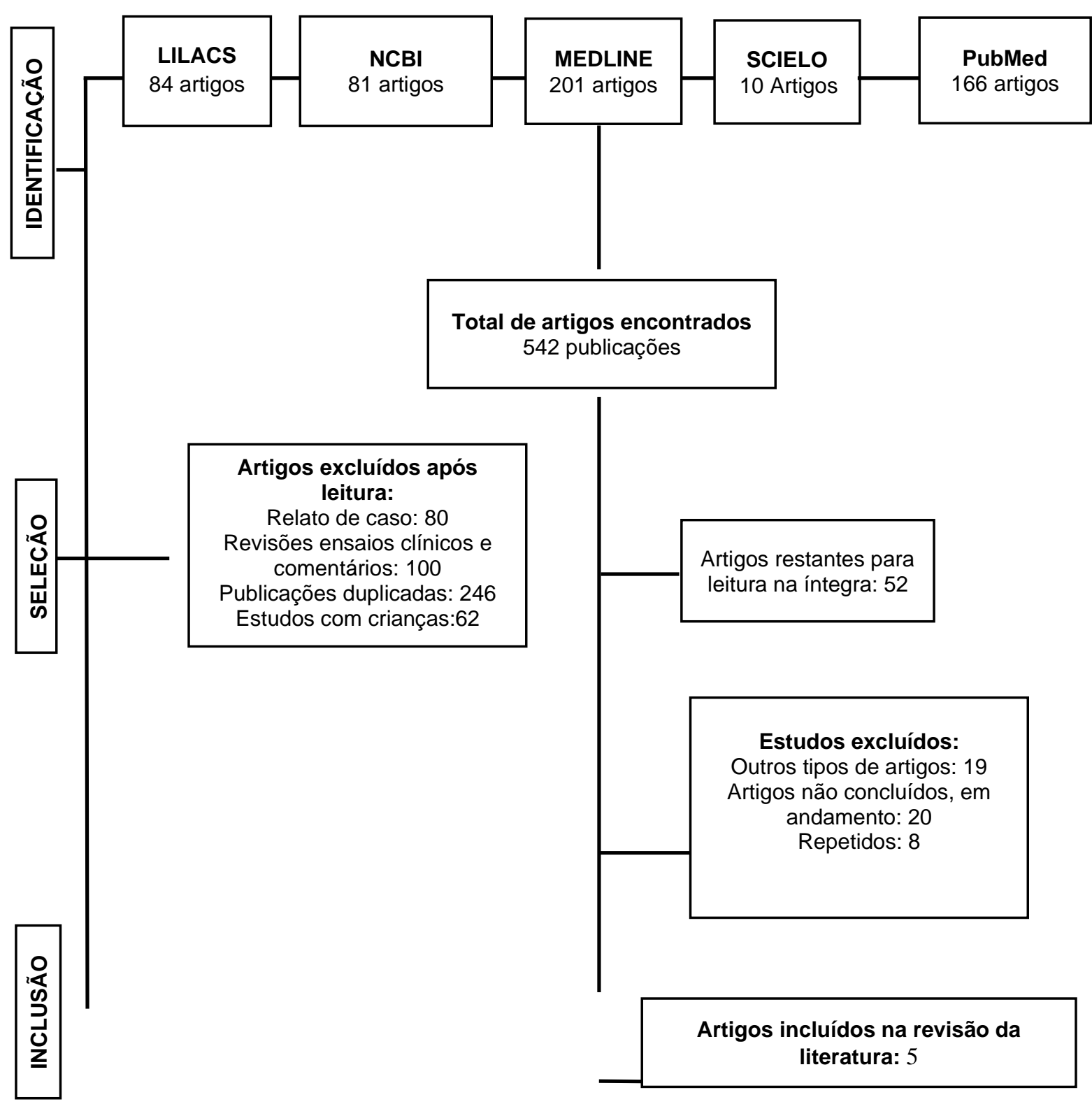




\begin{abstract}
O Brasil figura, em 2018, a segunda faixa mais alta de incidência de câncer de mama entre os todos os países, com uma taxa de 62,9 casos por roo mil mulheres (taxa padrão utilizada mundialmente) ...[...]... no Brasil um dos desafios é a redução das desigualdades entre as regiões e classes sociais. A mortalidade por câncer de mama está ligada, principalmente, ao acesso a diagnóstico e ao tratamento adequado no tempo oportuno. O objetivo é diagnosticar o câncer o mais precocemente possível, ainda nos estágios iniciais da doença, quando o tratamento é mais efetivo ...[...]... a desigualdade regional e social também se evidencia no acesso ao exame de mamografia de rastreamento, que deve ser realizado a cada dois anos por todas as mulheres na faixa etária de 50 a 69 anos. O percentual de mulheres brasileiras nessa faixa que fizeram o exame em 2013, de acordo com a última Pesquisa Nacional de Saúde, foi de $60 \%$, mas de apenas $38,7 \%$ na região Norte e $47,9 \%$ no Nordeste, bem abaixo das regiões Sul $(64,5 \%)$ e Sudeste (67,9\%). (INCA, 2019, p. I).
\end{abstract}

Vale enfatizar que, na grande maioria dos casos, o câncer de mama é decorrente de deformidades do material genético celular, as quais podem advir de anomalias genéticas hereditárias e/ ou adquiridas (por meio de erros de replicação celular) ou de atos como tabagismo, alcoolismo, obesidade, radiação, uso de substâncias químicas e utilização de agentes infecciosos (COELHO, 2014; MARTINEZ, 2015; OPAS, 2018; INCA, 2019; ACRUX et al., 2020;).

Caponero (2013), Acrux e colaboradores (2020) e INCA (2020), entendem ser indispensável ressaltar que que existem diversos "tipos" de câncer de mama, os quais podem se desenvolver em diferentes partes da mama; sendo referidos cânceres, divididos em: (I) câncer de mama não invasivo (carcinoma in situ) - encontrado nos ductos da mama (carcinoma ductal in situ ou DCIS) sem ter se espalhado para o tecido mamário ao redor dos ductos. O câncer de mama não invasivo é, comumente, encontrado durante uma mamografia e raramente aparece como um nódulo na mama, e (2) câncer de mama invasivo - onde as células cancerígenas se espalham através do revestimento dos ductos para o tecido mamário circundante. Este é o tipo mais comum de câncer de mama.

Os mAbs tem a capacidade de se ligar ao fator de crescimento endotelial vascular (VEGF), tornando a vascularização do tumor mais normal bem como levando o VEGF a seguir incapaz de ativar o receptor VEGF, que estimula o crescimento de novos vasos sanguíneos; atos esses que permitem que o tumor siga sendo nutrido bem como possibilita que medicamentos quimioterápicos possam chegar ao mesmo com uma maior eficácia, 
ajudando assim a parar e/ ou retardar o crescimento descontrolado e a disseminação do tumor (ZIDAN, 2020).

Tabela I. Resultados dos estudos selecionados

$\begin{array}{lll}\text { AUTORES TÍTULO } & \text { OBJETIVOS } & \text { PRINCIPAIS ACHADOS }\end{array}$

$\begin{array}{lll}\text { TORRES et al } & \begin{array}{l}\text { A promissão dos anticorpos } \\ \text { monoclonais }\end{array} & \text { Mostrar o benefício e } \\ \text { (2018) } & \text { ferramenta na farmacoterapia } & \text { vantagens dos anticorpos } \\ & \text { do câncer } & \text { importonais além de sua } \\ & \text { medicamentos usados na } \\ & \text { terapia do câncer. }\end{array}$

BRUNETO et Anticorpos monoclonais no al. (2019)

VIDAL;

FIGUEIREDO ; PERE (2018)

$\begin{array}{ll}\text { SANTANA et } & \begin{array}{l}\text { Principais tratamentos } \\ \text { utilizados no combate ao }\end{array} \\ \text { al. (2019) } & \begin{array}{l}\text { câncer de mama: uma revisão } \\ \text { de literatura }\end{array}\end{array}$

Identificar, na literatura, como manejar reações infusionais relacionadas aos anticorpos Oncológico: revisão de literatura para o Atendimento ao paciente e manejo das reações infusionais

O mercado brasileiro de anticorpos monoclonais utilizados para o tratamento de câncer registro sanitário ativo, no
Monoclonais; descrever protocolo de conduta para atendimento a reação infusional; identificar os anticorpos monoclonais de maior ocorrência de reações infusionais.

Identificar as principais características dos anticorpos monoclonais, destinados ao tratamento de câncer, com Brasil, em 2016.

Apresentar uma revisão de literatura sobre os principais métodos de tratamento do implicações e resultados. câncer de mama, suas
Apesar do custo com anticorpos monoclonais ser uma limitação para seu uso, representam uma das expectativas para o sucesso no tratamento do câncer e, à medida que sua eficiência ficar comprovada, sua produção poderá se estender, o que facilitará o acesso aos mesmos.

A segurança na implementação da administração intravenosa dos $\mathrm{mAbs}$, se encontra condicionada à estabilidade e compatibilidade dos fármacos

Foram identificados sete antígenos-alvos diferentes, sendo os principais os seguintes: EGFR, $\mathrm{CD}_{20}$ e HER2. No que diz respeito às indicações clínicas para as quais esses biológicos eram direcionados, observou-se uma maior frequência de linfomas, leucemias e também de cânceres de mama, cólon e reto.

As recomendações gerais indicam essencialmente terapia hormonal isolada para pacientes com câncer de mama "Luminal A" e terapia hormonal acompanhada de quimioterapia para pacientes acometidos do subtipo "Luminal B”. Para pacientes com câncer de mama "HER2-positivo" a recomendação é de terapia 
hormonal, quimioterapia, além de terapia-alvo anti-HER2, enquanto para a maioria dos pacientes com doença "triplo-negativa", é recomendada quimioterapia.

$\begin{array}{ll}\text { CORDEIRO et } & \text { ANTICORPOS } \\ \text { al (2014) } & \text { MONOCLONAIS: } \\ & \text { IMPLICAÇÕES } \\ & \text { TERAPÊUTICAS } \\ & \text { CÂNCER }\end{array}$

\begin{tabular}{ll} 
Buscou-se & \multicolumn{2}{c}{ identificar os } \\
anticorpos monoclonais \\
disponíveis no mercado e \\
NO descrever seus aspectos \\
farmacológicos.
\end{tabular}

O uso de fármacos a base de biomoléculas recombinantes vem surgindo como uma promissora e possível realidade no tratamento de doenças, de forma mais específica e eficaz

Diante do cenário contemporâneo alarmante do câncer de mama, não só no Brasil como em todo o mundo, diversas pesquisas clínicas têm buscado inovações, em especial, junto ao tratamento desses, sendo as mais promissoras e positivas as que envolvem o emprego de tecnologias citotóxicas inovadoras a inibir a angiogênese, tais quais os mAbs (CARVALHO, 2013).

Por isso tem-se que inibidores de angiogênese, tais como os inibidores monoclonais (mAbs), podem ser deveras positivos ao tratamento de certos tipos de câncer, dentre eles o de mama, uma vez que esses atuam seletivamente sob os vasos sanguíneos que alimentam os tumores, melhorando a circulação dos existentes e impedindo a formação de novos, o que além de interromper o crescimento das células cancerígenas melhora a "chegada" de quimioterápicos ao tumor, o que, indiretamente, "mata" o mesmo (SILVA et al., 2or6; FENG et al., 2018; ZIDAN, 2020).

\section{CONCLUSÃO}

A terapia envolvendo a aplicação de mAbs no tratamento do câncer de mama é satisfatório, pois apresenta menor risco de reações adversas, além de melhor eficácia no tratamento da patologia. No entanto, um melhor conhecimento sobre as reações infusionais e o seu mecanismo de ação são necessários. Mesmo que o custo relacionado a anticorpos possa ser uma limitação para a utilização, a sua produção poderá sofrer ampliação, o que possibilitaria maior acessibilidade ao tratamento. Além disso, outros estudos que aprimore pacientes com câncer de mama submetidos à terapêutica com mAbs são necessários no sentido de elucidar os questionamentos sobre os mecanismos que envolvem o processo de resistência ao tratamento químico destas células. 


\section{REFERÊNCIAS}

ACRUX, T.; ATHANAZIO, D.; GAUDÊNCIO, D.; ROCHA, C. Carcinoma ductal in situ da mama: correlação dos achados arquiteturais, citológicos e IHQ e análise de recorrência. Jornal Brasileiro de Patologias e Medicina Laboratorial. 2020; vol.56. (https://www.scielo.br/pdf/jbpml/v56/pt_1676-2444-jbpml-56-e1742020.pdf).

(https://doi.org/ro.5935/1676-2444.20200018).

ALVES, P. C.; SOUSA, A. P.; SANTOS, M. C. L.; FERNANDES, A. F. C. Conhecimento e expectativas de mulheres no pré-operatório da mastectomia. Revista Escola de Enfermagem- USP. 2ог. (https://www.scielo.br/pdf/reeusp/v44n4/19.pdf). https://doi.org/10.1590/Soo8o-62342010000400o19

BRUNETO, R. V.; ARRUDA, G. N.; FERNANDES, K.T.; PACCA, F.C.; VEIGA,T.; Anticorpos monoclonais no tratamento oncológico: revisão de literatura para o atendimento ao paciente e manejo das reações infusionais. Archives of Health Sciences. 2019 Out-Dez 26(3) 173-178. https://doi.org/10.17696/2318-3691.26.3.2019.1369

CARVALHO, APF. Estudos clínicos e patentes de anticorpos monoclonais para o tratamento do câncer [Dissertação de Mestrado]. Rio de Janeiro: Instituto de Tecnologias em Imunobiológicos, Fundação Oswaldo Cruz; 2013.

CAPONERO, R. Guia de câncer de mama. $5^{\circ}$ ed. São Paulo: MG editores, 2013. 13op.

COELhO, J. T. A. Anticorpos Monoclonais. 2014, 9if. Mestrado (Ciências Farmacêutica). Porto: Universidade Fernando Pessoa, 2014.

CORDEIRO, M. L.S.; SILVA, N.L.F.; VAZ, M.R.F.; NOBRÉGA, F.F.F. ANTICORPOS MONOCLONAIS: IMPLICAÇÕES TERAPÊUTICAS NO CÂNCER. Revista saúde e ciência On line, 2014; 3(3):252-262, set-dez, 2014. (https://doi.org/ro.35572/rsc.v3i3.329).

FENG, Y.; SPEZIA, M.; HUANG, S.; YUAN, C.; ZENG, Z.; ZHANG, L.; LIU, W.; HUANG, B.; LUO, W.; LIU, B.; LEI, Y.; DU, S.; VUPPALATI, A.; LUU, H. H.; HAYDON, R.C.; HE, T.C.; REN, G. Breast Cancer Development and Progression: Risk Factors, Cancer Stem Cells, Signaling Pathways, Genomics, and Molecular Pathogenesis. Genes Dis 2018; 5 (2), 77-106. (https://doi.org/ro.1016/j.gendis.2018.05.001).

FIGUEIREDO, A. P. Diferenças entre tumores benignos e malignos. Publicado oo5/04/2013. Disponível em: http://stopcancerportugal.com/2013/04/o5/diferencas-entre-tumoresbenignos-e-malignos/. Acesso em II/o9/2020. 
FONTOURA, B. A.; CAIXETA, E. S.; SILVA, L. S.; SILVA, R. G. C; PEREIRA, V. C. B.; PASSOS, M. A. N. Imunoterapia como tratamento de câncer e o papel da enfermagem. Research, Society and Development, v. io, n. 6, e38710615902, 2021. (https://doi.org/ro.33448/rsd-vioi6.15902).

INCA. Câncer de mama. S/ d. disponível em: https://incariopreto.com.br/cancer-demama/. Acesso em Io/04/2021.

JORGE, J. J. Imunoterapia no tratamento do câncer. Arquivos de Asma, Alergia e Imunologia. 2019;3(2):133-138. Doi: 10.5935/2526-5393.20190023.

LEAL, J. H. S.; CUBERO, D.; GIGLIO, A. D. Hormônio terapia paliativa em câncer de mama: aspectos práticos e revisão da literatura. Revista Brasileira de Clínica Medica. 2oro; pag.338-343. (http://files.bvs.br/upload/S/1679-1010/2010/v8n4/aoio.pdf).

MACEDO, M. J. P. B. Estratégias e inovações aplicadas ao desenvolvimento de anticorpos monoclonais. Trabalho de Conclusão do Curso de Farmácia-Bioquímica da Faculdade de Ciências Farmacêuticas da Universidade de São Paulo. São Paulo, 2018.

MARQUES, C. H. Aspectos fundamentais à implantação da tecnologia de produção de anticorpos monoclonais humanizados com potencial aplicação terapêutica. Tese (Mestrado). Instituto Oswaldo Cruz, Biologia Celular e Molecular. 2005.

MARTINEZ, A. T. A. Intervenção Educativa nas mulheres com fatores de risco de câncer de mama na UBS Misael Pinto dos Santos em Pedra do Indaiá - MG. 2015, 6of. Trabalho de Conclusão de Curso (Especialista em Estratégia de Saúde da Família). Pedra do Indaiá: UFMG, 2015 . (https://www.nescon.medicina.ufmg.br/biblioteca/imagem/interven\% $\mathrm{C}_{3} \% \mathrm{~A}$ 7ao_educati va_nas_mulheres.pdf).

NASCIMENTO, A. S.; MELLO, E. V. S. L.; SCHNEIDER, L. C. L.; AlMEIDA, F. L. A. Principais tratamentos utilizados no combate ao câncer de mama: uma revisão de literatura. Arquivos do MUDI, v 23, n 3, p. 20I-219, 2019. (https://doi.org/I0.4025/arqmudi.v23i3.51538).

OPAS. Folha informativa: Câncer. Publicado setembro 2018. Disponível em: https://www.paho.org/bra/index.php?option=com_content\&view =article\&id=5588: folhainformativa-cancer\&Itemid=1094. Acesso II/o9/2020.

PEÑA, Y.; PERERA, A.; BATISTA, J. F. Immunoscintigraphy and radioimmunotherapy in Cuba: experiences with labeled monoclonal antibodies for cancer diagnosis and treatment (1993-2013). MEDICC Review. 2014; 16 (3-4): 55-6o. (https://www.scielosp.org/article/medicc/2014.vi6n3-4/55-6o/). 
SANTANA, A. N.; MELLO, E.V.S.L.; SCHNEIDER, L.C.L.; ALMEIDA, F.L.A. Principais tratamentos utilizados no combate ao câncer de mama: uma revisão de literatura. Arquivos do mudi [Internet]. 19을 de dezembro de 2019 [citado $3^{\circ}$ de junho de 2021];23(3):20119. Disponível em: https://periodicos.uem.br/ojs/index.php/ArqMudi/article/view/51538. (https://doi.org/10.4025/arqmudi.v23i3.51538)

SANTOS, R. V., LIMA, P. M. G.; NITSHE, A.; HARTH, F. M.; MELO, F. Y.; AKAMTSU, H.T.; LIMA, H. C. Aplicações Terapêuticas dos anticorpos Monoclonais. Revista Brasileira Alergia e Imunopatologia, Vol. 29, nº 2, 2006.

SANTOS, B. S. Anticorpos monoclonais no combate de células tumorais: uma revisão bibliográfica. Revista Acadêmica, edição2ı. Disponível em:<https://oswaldocruz.br/revista_academica/content/pdf/Edicao_2I_BRUNA_DE_SO UZA_DOS_SANTOS.pdf $>$. Acesso em junho de 2021.

SILVA, C. F.; SILVA, M. V.; OSORIO-DE-CASTRO, C. G. S. Os ensaios clínicos e o registro de anticorpos monoclonais e biomedicamentos oncológicos no Brasil. Revista Panamericana de Salud Publica. 39 (3) Mar 2016. Disponível em: https://www.scielosp.org/article/rpsp/2016.v39n3/149-156/

TORRES LV et al. A Promissão dos Anticorpos Monoclonais como Ferramenta na Farmacoterapia do Câncer. Revista Saúde e Ciência online, v. 7, n. 2, (maio a agosto de 2018). 502 p. DOI https://doi.org/I0.35572/rsc.v7i2.96

VIDAL, TJ; FIGUEIREDO, T; PEPE, VL. O mercado brasileiro de anticorpos monoclonais utilizados para o tratamento de câncer. Cadernos de Saúde Pública [online]. 2018, v. 34, n. I2 [Acessado3 Junho 2021] , eooorogr8. Disponível em: 〈https://doi.org/ro.1590/oro2-3irXoooro918〉. Epub 29 Nov $2018 . \quad$ ISSN I678-4464. https://doi.org/I0.1590/oro2-311Xooorogi8.

ZIDAN, O. H. PEGylated Chitosan/ Doxorubicin Nanoparticles and conjugated with Monoclonal Antibodies for Breast Cancer Therapy. 2020, I56f. Tese (Mestrado em Ciências). Cairo: The American University in Cairo, 2020. (http://dar.aucegypt.edu/bitstream/handle/10526/5859/Turnitin\%20\%282\%29\%20.pdf?seq uence $=3$ ).

WHO. Brazil Source: Globocan 2018. Publicado maio 2019. Disponível em: https://gco.iarc.fr/today/data/factsheets/populations/76-brazil-fact-sheets.pdf. Acesso Io/04/202I. 